\title{
On 3D flow-structures behind an inclined plate
}

\author{
Václav Uruba ${ }^{1,3, *}$, David Pavlík $^{2}$, Pavel Procházka ${ }^{1}$, Vladislav Skála ${ }^{1}$ and Václav Kopecký ${ }^{2}$ \\ ${ }^{1}$ Institute of Thermomechanics, AS CR, v.v.i., Dolejškova 5, Praha 8, Czechia \\ ${ }^{2}$ Institute of New Technologies, Technical University of Liberec, Studentská 2, Liberec, Czechia \\ ${ }^{3}$ University of West Bohemia, Faculty of Mechanical Engineering, Universitní 22, Plzeň, Czechia
}

\begin{abstract}
Stereo PIV measurements has been performed behind the inclined plate, angle of attack 5 and $10 \mathrm{deg}$. Occurrence and dynamics of streamwise structures behind the plate trailing edge have been studied in details using POD method. The streamwise structures are represented by vortices and low- and highvelocity regions, probably streaks. The obtained results support the hypothesis of an airfoil-flow force interaction by Hoffman and Johnson [1,2].
\end{abstract}

\section{Introduction}

The motivation of the presented study is supporting new ideas about principle of flight by Hoffman and Johnson from KTH Stockholm, see [1,2]. The new hypothesis of physical mechanism of flight relies on existence of streamwise vortical structures on the suction side of the airfoil and within its wake. The vortices origin is supposed to be the instability of the boundary layer subjected to adverse pressure gradient on the airfoil suction side (i.e. upper) - see [3]. Some consequences of the hypothesis are shown in $[4,5,6]$.

The simple experiment is made with the simplest airfoil possible represented by a flat plate in uniform flow and moderate angle of attack. However the experimental results are of a qualitative nature only, they reveals existence of streamwise vortices above the plate without any doubt.

\section{Experimental setup}

Flat plate inclined with angle of attack 5 and 10 degs has been placed in a uniform low turbulence stream. The blow-down facility produces a jet with uniform velocity distribution, mean velocity about $5 \mathrm{~m} / \mathrm{s}$, intensity of turbulence less than $0.2 \%$. The plate of thickness $2 \mathrm{~mm}$ had rounded edges, chord $100 \mathrm{~mm}$ and span $300 \mathrm{~mm}$.

As for the measuring technique, the time-resolved stereo PIV method was used for the experiments. The measuring system DANTEC consists of laser with cylindrical optics and two CMOS cameras. The software Dynamics Studio 3.4 was used for velocity-fields evaluation. Laser New Wave Pegasus Nd:YLF, double head, wavelength $527 \mathrm{~nm}$, maximal frequency $10 \mathrm{kHz}$, a shot energy is $10 \mathrm{~mJ}$ for $1 \mathrm{kHz}$ (corresponding power $10 \mathrm{~W}$ per head). Two cameras NanoSense MkIII, resolution $1280 \times 1024$ pixels and frequency 500 and 800 double-snaps per second, 1000 double-snaps were acquired in sequence corresponding to $2 \mathrm{~s}$ of the record time. The stereo-PIV method has been used for evaluation all 3 velocity components in the measuring plane.

The measuring plane has been located perpendicular to the main flow, $35 \mathrm{~mm}$ behind the plane trailing edge covering the span $60 \mathrm{~mm}$ in the flow-field middle position. The situation is shown in Fig. 1.

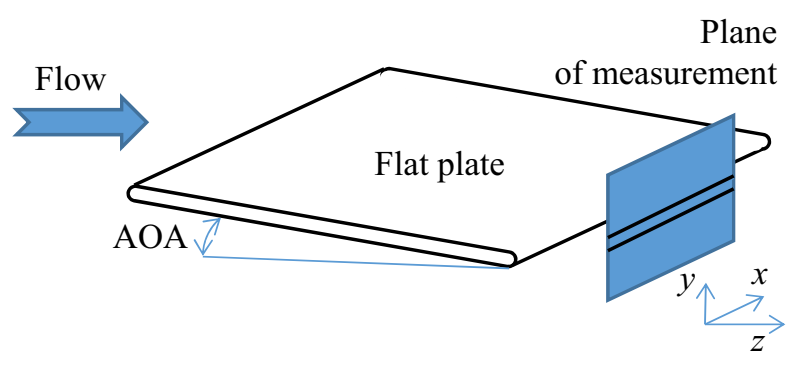

Fig. 1. Schematics of the experiment.

In Fig. 1 the Cartesian coordinate system is introduced, plane $x y$ is the measuring plane, mean flow is in the $z$ direction. AOA stands for angle of attack.

\section{Results}

Two variants are considered characterized by the angle of attack 5 and 10 degrees.

In Fig. 2 three instantaneous velocity fields arbitrarily chosen are shown, black vectors represent the projection of velocity vectors to the $x y$ measuring plane, colour denotes the third velocity component $w$ in the $z$ direction. The two horizontal white lines (for $y=0$ and $2 \mathrm{~mm}$ ) represent projection of the plate trailing edge. To visualize the velocity field structure, the vector-lines are added arbitrarily in white.

\footnotetext{
Corresponding author: uruba@it.cas.cz
} 
The 3 consecutive snapshots are presented in Fig. 2 with double acquisition period.
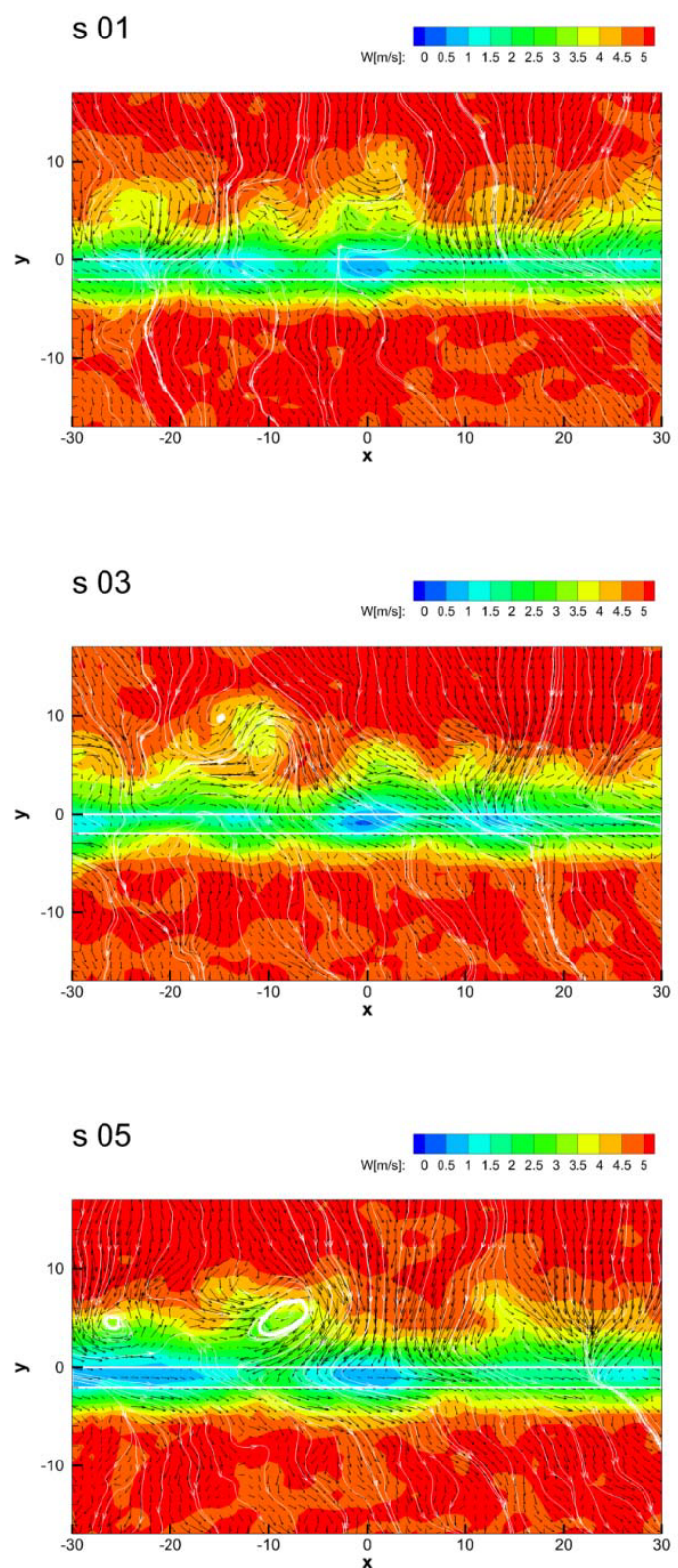

Fig. 2. 3 snapshots, angle of attack $5 \mathrm{deg}$.

The flow seems to be perturbed by presence of the plate, behind the plate trailing edge the low-velocity wake is visible. The flow disturbances in the upper half (i.e. above the plate) are of character vortices, while flow below the plate is more regular.

Similar conclusions could be made from the instantaneous velocity fields generated by the plate with angle of attack $10 \mathrm{deg}$ in Fig. 3. Wake is in this case much bigger, the location of minimum velocity is a little bellow the trailing edge position due to the distance of the measuring plane. In this case the flow above the plate is much more perturbed, the flow bellow is regular, but deflected downwards due to the plate downwash effect.
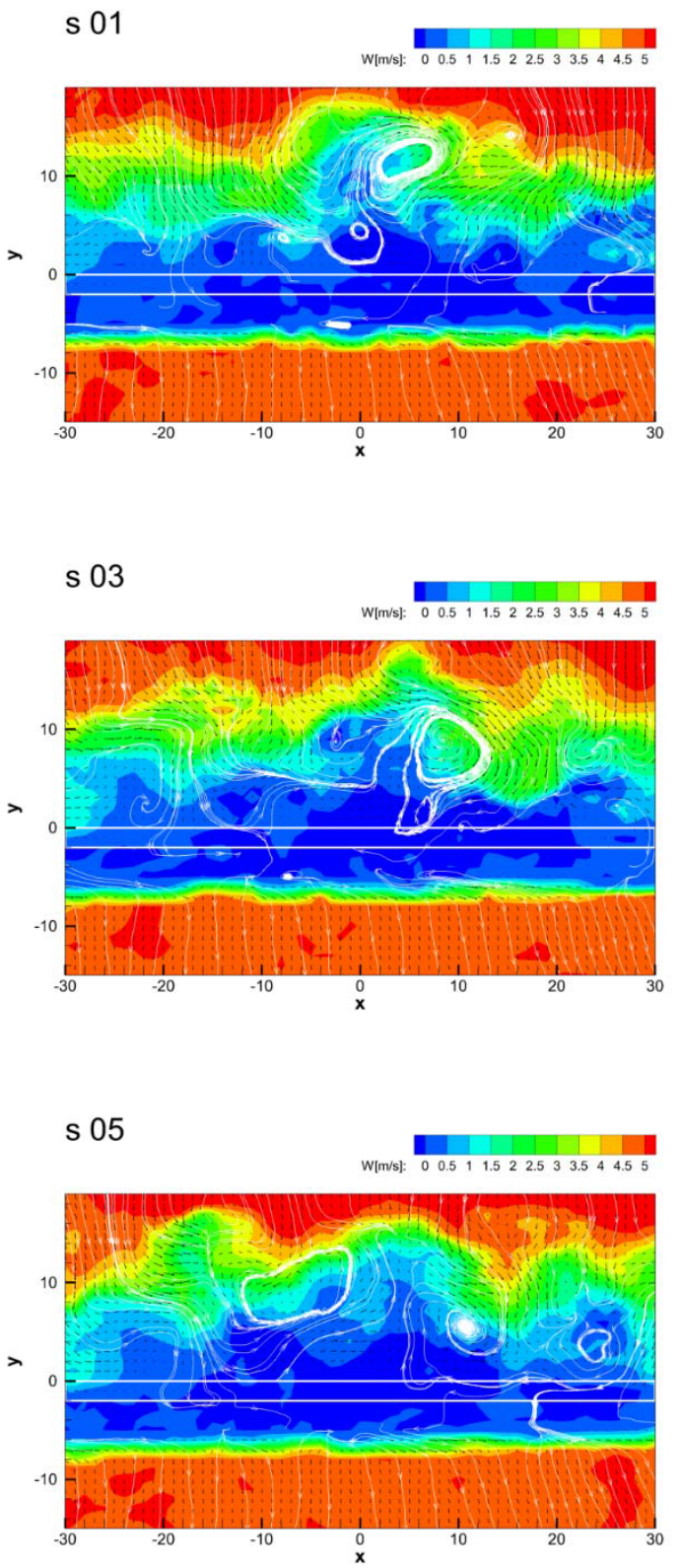

Fig. 3. 3 snapshots, angle of attack $10 \mathrm{deg}$.

The high-frequency dynamical behaviour of the flow above the plate is visible, as the flow patterns differ considerable from each other.

\subsection{Mean flow picture}

First, the statistical characteristics of the flow have been evaluated. In Fig. $4 \mathrm{a}$ and $4 \mathrm{~b}$ the time-mean velocity field is shown for angle of attack $5 \mathrm{deg}$ and $10 \mathrm{deg}$ respectively. The low-velocity wake is located behind the trailing edge, little bellow it due to downwash effect. The wake is much more pronounced for the $\mathrm{AOA}=10 \mathrm{deg}$, it is well extended above the plate. 


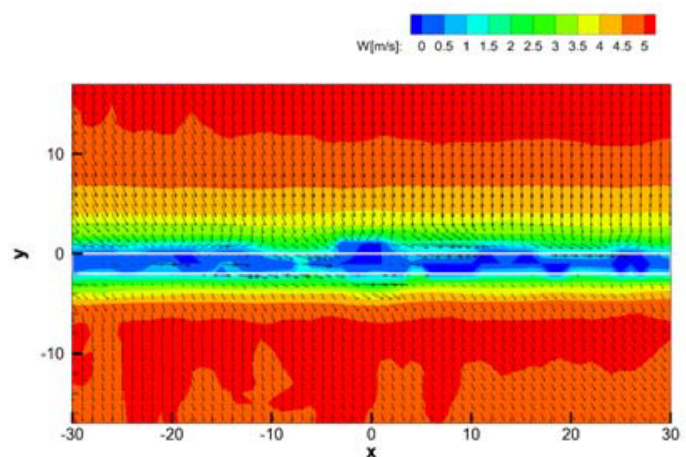

(a)

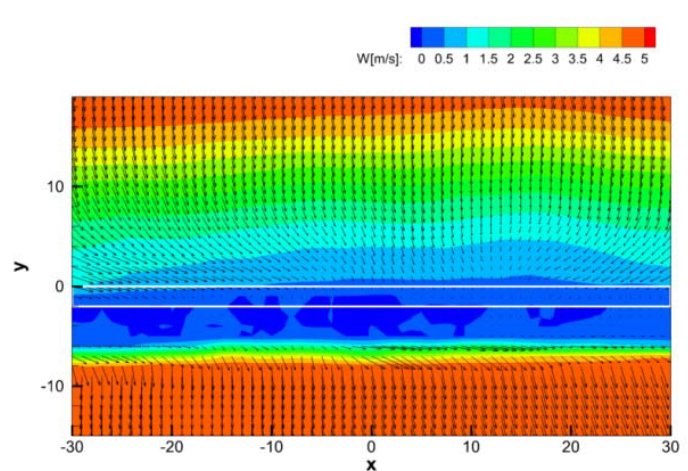

(b)

Fig. 4. Time-mean velocity distributions for angle of attack $5 \mathrm{deg}$ (a) and $10 \mathrm{deg}$ (b)

Please note that no vortical structures are detectable in the time-mean velocity field topology.

The dynamical activity could be characterized by velocity variance. In Fig. $5 a$ and $5 b$ there are distributions of sums of all 3 velocity components variances. The scale for both cases is the same, it is clear that the maximal value of the sum of velocity components variance is for the angle of attack $10 \mathrm{deg}$ more than one order of magnitude higher than for $5 \mathrm{deg}$.

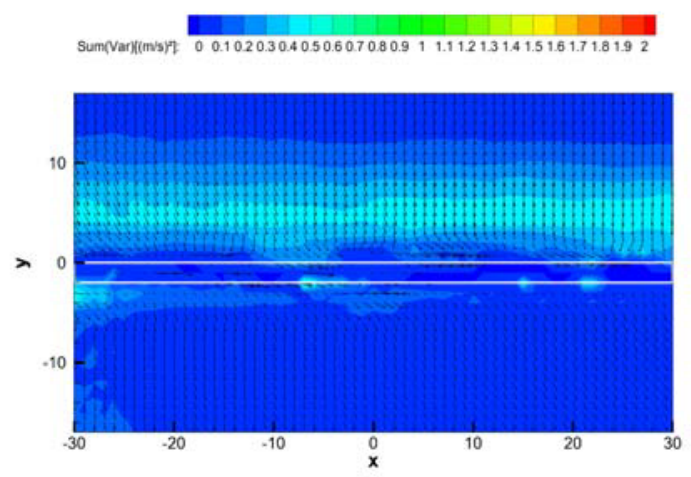

(a)

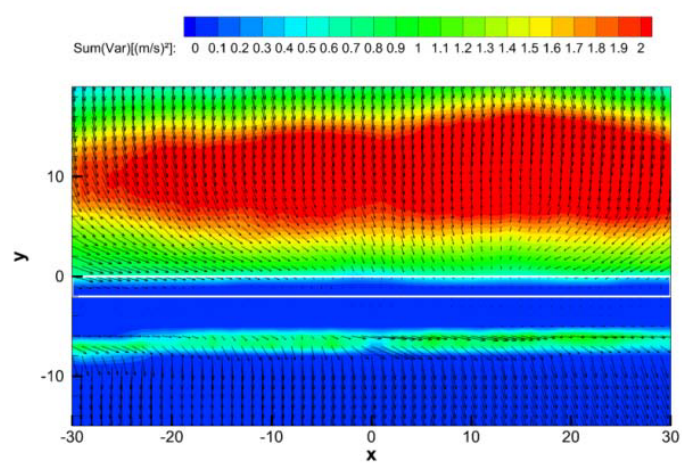

(b)

Fig. 5. Sum of velocity components variance distributions for angle of attack 5 deg (a) and $10 \operatorname{deg}($ b)

Maximum of dynamical activity is located about $5 \mathrm{~mm}$ above the trailing edge in the case of $\mathrm{AOA}=5 \mathrm{deg}$, while for $\mathrm{AOA}=10 \mathrm{deg}$ the maximum is located $10 \mathrm{~mm}$ above the plate trailing edge.

\subsection{Flow dynamics}

The flow-field dynamics could be quantified from velocity variances distribution in Fig. 5 . Here we could distinguish locations with low dynamical activity (deep blue) and higher dynamical activity (green, yellow) and highest (red).

To study the topology dynamics in details, the Proper Orthogonal Decomposition method (hereinafter POD) has been applied to the vector-field snaps. The POD method indicates the topological orthogonal modes containing maximum fluctuation energy (i.e. velocity fluctuation variance). More details on the POD method see e.g. $[7,8]$.

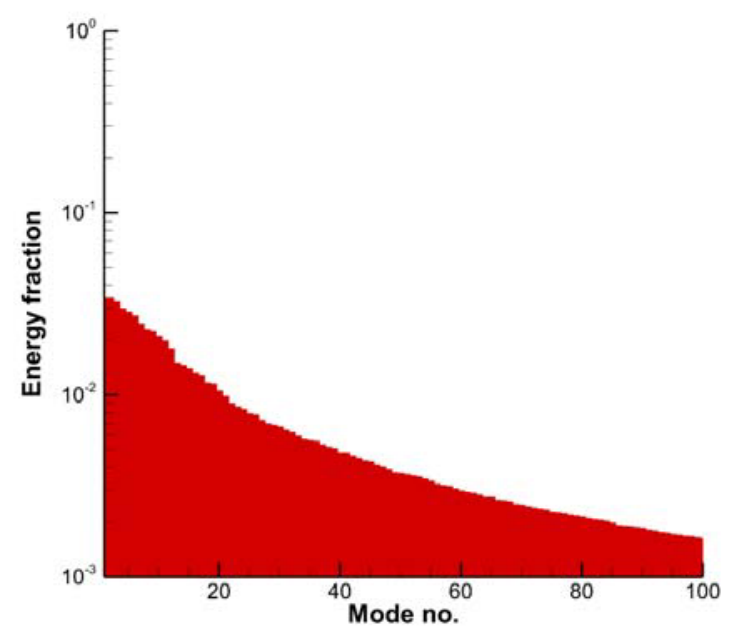

Fig. 6. Energy fraction of the POD modes, angle of attack $5 \mathrm{deg}$. 
First the case of angle of attack 5 deg has been analysed, the POD modes have been computed. The POD modes are ordered according to theirs energy beginning from POD 1 with the highest energy. In Fig. 6 there is energy fraction of first 100 POD modes, the most energetic mode POD 1 contains about $3.5 \%$ of the total energy of velocity fluctuations only.

The topology of POD modes will be presented in the form of vector-distribution patterns, only in-plane velocity components are to be shown. Colour denotes the streamwise velocity component $w$ in $z$ direction, red colour means positive velocity fluctuation (i.e. high velocity region), while blue colour means negative velocity fluctuation (i.e. low velocity region). As a vortex is typical element of the velocity field, the swirling strength has been used for identification. Swirling strength is defined as the imaginary part of the complex eigenvalue of the velocity gradient tensor. Local minima of negative-valued swirling strength indicate a vortex core position, while positive values indicate areas of the flow, where shear may be present, but no swirling motion. We will show isolines of negative-valued swirling strength in green, typically forming closed curves around a vortex core. Centre of a vortex could be considered as the centre of the structure, number of isolines indicates the vortex strength (more isolines, higher vortex strength).

In Fig. 7 there are a few selected POD modes with typical topologies. The position of the plate trailing edge is indicated by black lines.
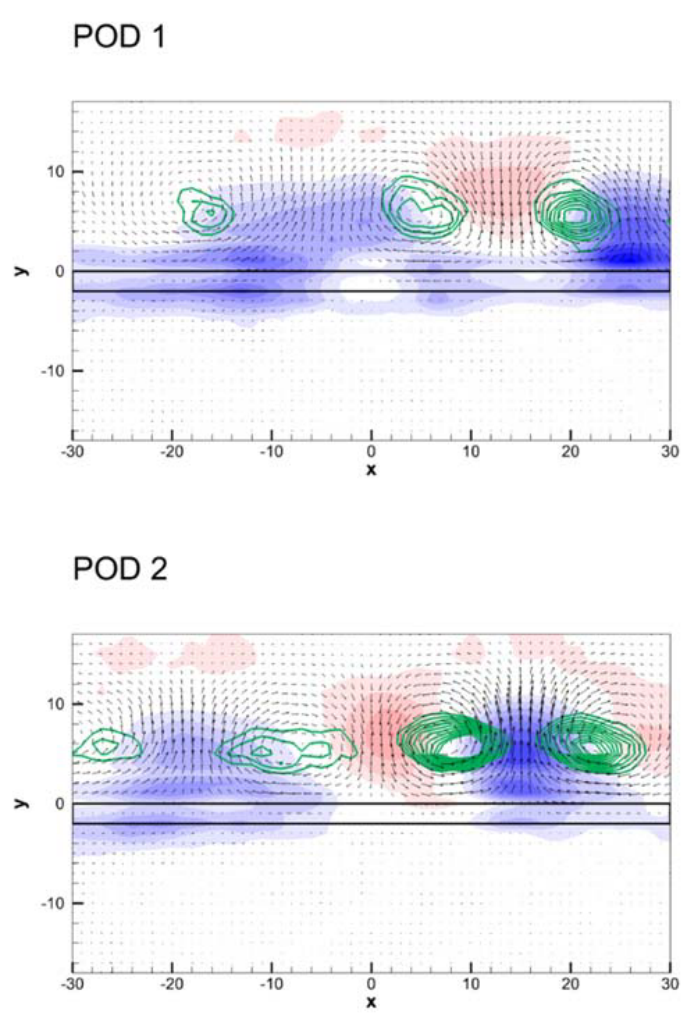

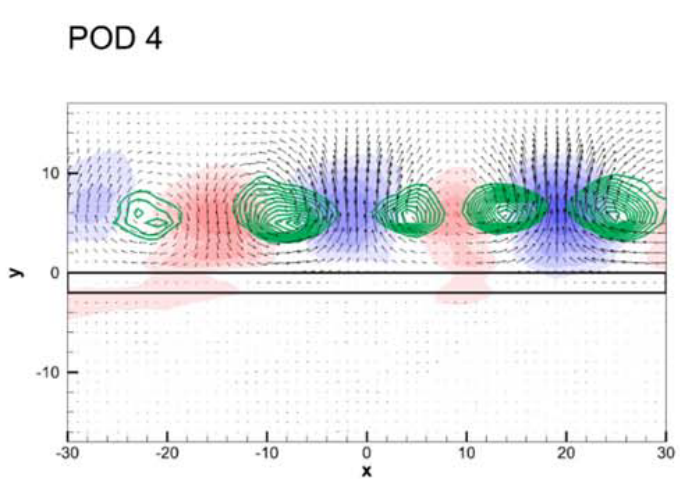

POD 11

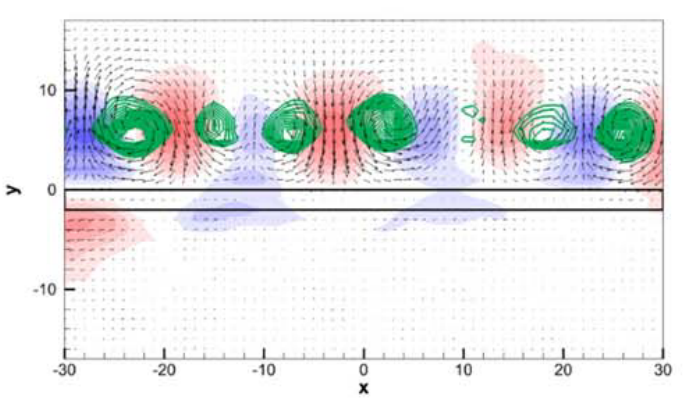

POD 21

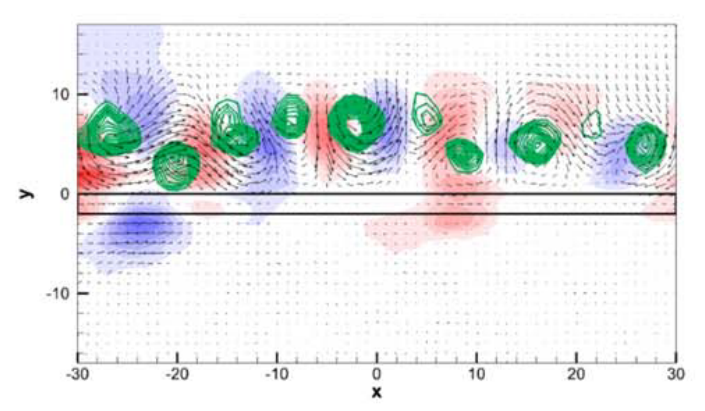

POD 58

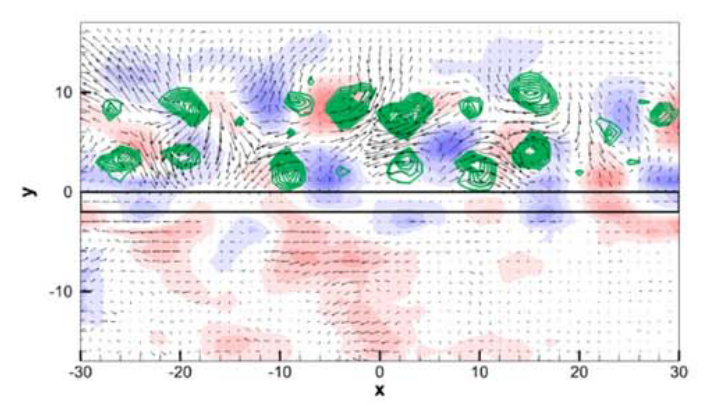

Fig. 7. Selected POD modes topologies, angle of attack 5 deg. 
The POD modes in Fig. 7 show generally system of vortices located along the trailing edge, above it. Typically the adjacent vortices are of opposite orientation, the space between the neighbouring vortices is filed by fluid of either higher or lower streamwise velocity regions. This could indicate low- and highvelocity streaks, however the analysis is provided only in one plane perpendicular to the flow, we have no information about topology in $z$ direction. The dynamical structures could be modulated also in the streamwise direction, or not. Anyway, the streamwise velocity component variations $w$ are determined by distribution of mean velocity component $v$ in vertical $(y)$ direction, see Fig. 4. Generally, if the fluid element is transported by the vortex motion from the region of higher mean velocity (on the top) to low velocity region (wake), the streamwise velocity fluctuation is positive and vice versa. It seems to be a rule for the fluctuation velocity components within the upper half of the pictures $(y>0)$ : for the positive spanwise fluctuation velocity component $v$ (upward vectors orientation), the fluctuation of streamwise velocity component $w$ is negative and for negative $v$ fluctuation velocity component (downward vectors orientation) the of streamwise velocity component $w$ is positive.

The high energy modes carry long streamwise structures, both vortices and streaks, while higher order (low energy) modes are more structured also in streamwise direction, very probably. The details should be subject of further research.

Anyway, it is clear that the high energy modes (low order POD modes) contain bigger structures than the low energy modes.

The same analysis has been carried out for the case of angle of attack $10 \mathrm{deg}$. In Fig. 8 there is energy fraction of first 100 POD modes, energy of the mode 1 is about $12.3 \%$ of the total energy of velocity fluctuations.

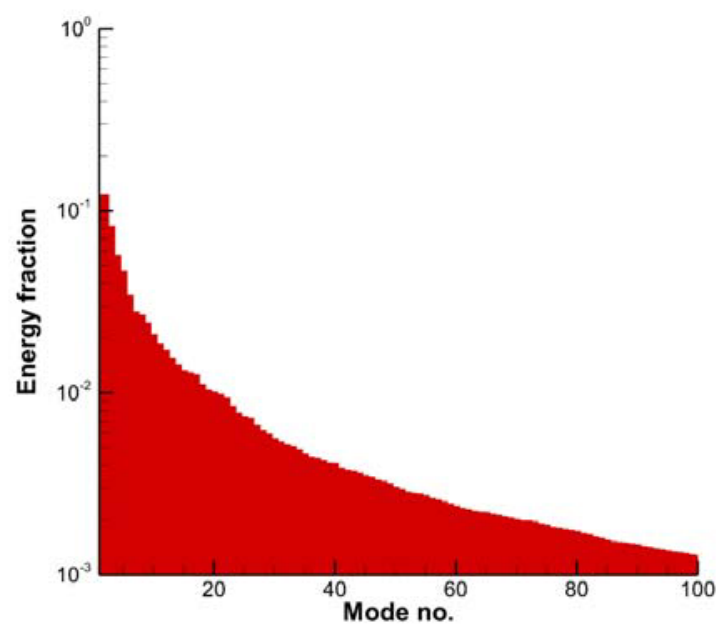

Fig. 8. Energy fraction of the POD modes, angle of attack $10 \mathrm{deg}$.
Selected POD modes topologies with typical vortical structures and streaks are in Fig. 9.

POD 1

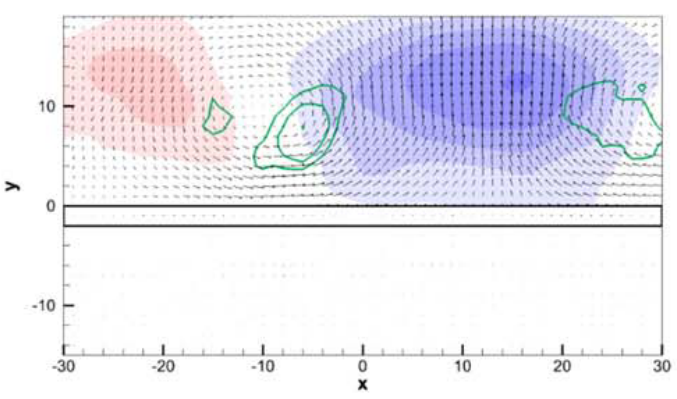

POD 4

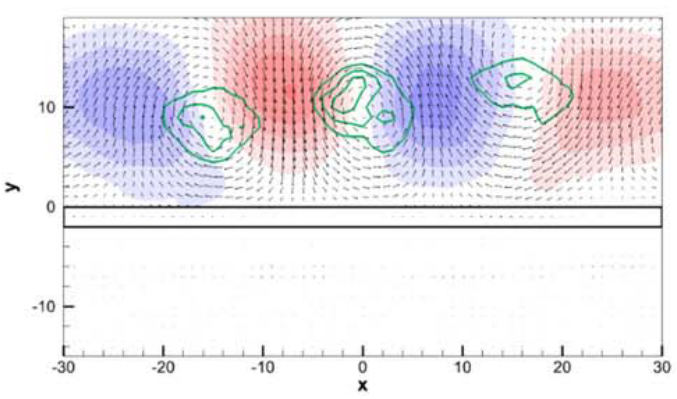

POD 6

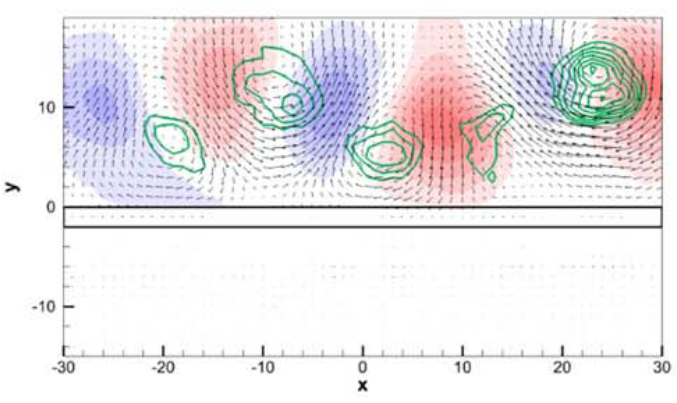

POD 15

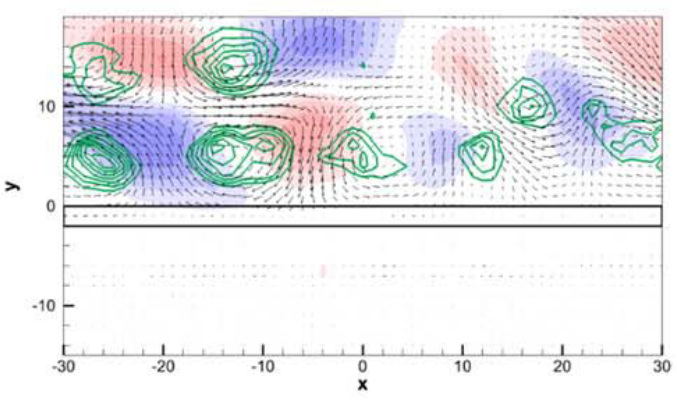




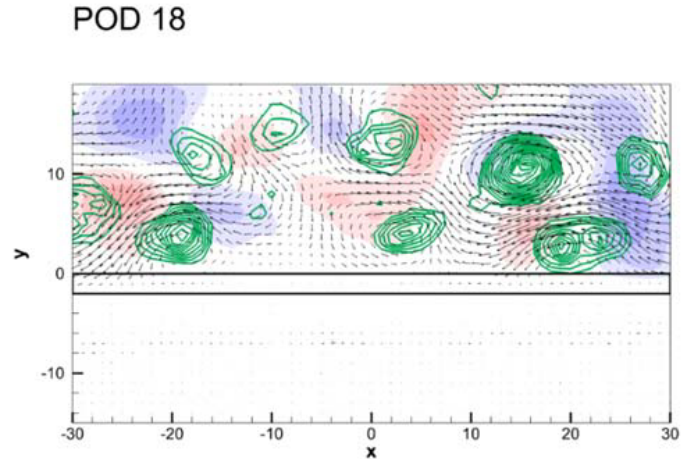

POD 37

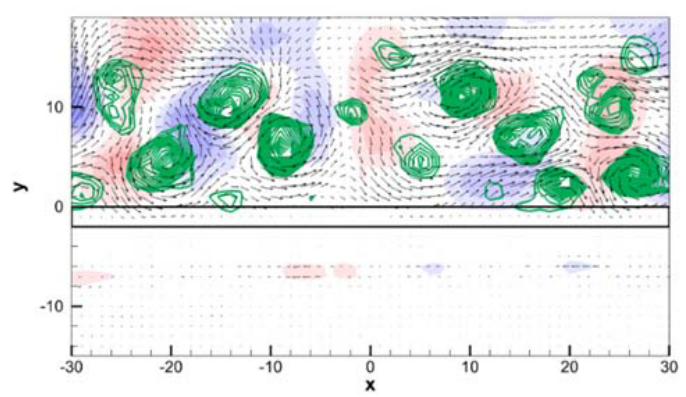

POD 89

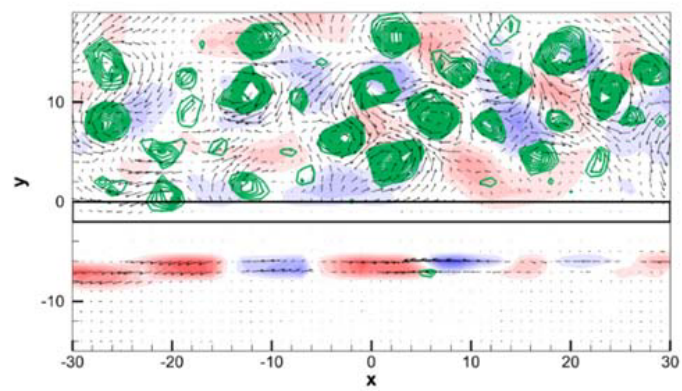

Fig. 9. Selected POD modes topologies, angle of attack $10 \mathrm{deg}$.

The observation of the POD modes topology is similar with the case of angle of attack $5 \mathrm{deg}$, however the touched area is of much bigger extend, especially in the upper part above the plate. The structures, both vortical and streaks, are distributed in more rows above the plate, for the higher order modes the space is completely filled by the structures (see e.g. POD mode 89). Lower order modes are characterized by a few number of very big structures in space (e.g. POD 1,4,6). For the lower order modes the vortices strength seems to be relatively week, however it should be noticed, that for the purpose of presentation the vector field is normalized and does not reflect the mode energy. Thus, the topology figures could provide qualitative information only.

\section{Conclusions}

The experimental results reveals existence of dynamical structures within the boundary layer on the plate suction side. Two types of structures are identified interacting with each other: streamwise vortices and low- and highvelocity regions, probably streaks. However experiments have been carried out in a single measuring plane behind the plate trailing edge, evolution of the dynamical structures should be studied in future.

The time-mean topology is vortex free.

The presented results support the theory of lift force generation by Hoffman and Johnson published in [1,2], however they could not be considered as the final and decisive confirmation.

\section{Acknowledgement}

The authors acknowledge the support of the Technology Agency of the Czech Republic by means of projects TA04020129 and TA04011437.

\section{References}

1. J. Hoffman, C. Johnson, The Mathematical Secret of Flight, Normat 57, 4, pp.1-25, (2009)

2. J. Hoffman, C. Johnson, Resolution of d'Alembert's Paradox, J. Math. Fluid Mech., Vol.12 (3), pp.321334, (2010)

3. P. Manneville, Instabilities, Chaos and Turbulence. (Imperial College Press, 2004)

4. V. Uruba, Force Interaction of an Airfoil in FluidFlow, 15th conference on Power System Engineering, Thermodynamics \& Fluid Flow - ES 2016, June 09 - 10, 2016, Pilsen, Czech Republic, 7375, 7p., (2016)

5. V. Uruba, On Aerodynamic Forces Physical Mechanism, SKMTaT 2016, 20-23 June, 2016, Slovak University of Technology in Bratislava (R. Olšiak, K. Prikkel), pp. 258-262 (2016)

6. V. Uruba, On 3D Instability of Wake behind a Cylinder, AEaNMiFMaE 2016, 27-29 April 2016, University of Žilina, Department of Power Engineering (R. Lenhard, K. Kaduchová) pp. 253256 (2016)

7. V. Uruba, Near Wake Dynamics around a Vibrating Airfoil by Means of PIV and Oscillation Pattern Decomposition at Reynolds Number of 65000 , Journal of Fluids and Structures, 55, pp. 372-383 (2015)

8. V. Uruba, Decomposition Methods in Turbulent Research, EFM11, EPJ Web of Conferences, 25 01095 (2012) 